\title{
Latest Developments in Cognitive Functioning in Mood and Anxiety Disorders
}

\author{
Felicia Gould $^{1}$ - Sara Kaplan ${ }^{1}$ Philip D. Harvey ${ }^{1,2}$
}

Published online: 9 July 2015

(C) Springer International Publishing AG 2015

\begin{abstract}
Cognitive impairments are well-documented features of mood and anxiety disorders. Recent developments in research on cognition, assessment techniques, treatments, and comorbidities in cognitive changes across major depression, bipolar disorder, and post-traumatic stress disorder (PTSD) are reviewed. Promising treatments have been reported but are tempered by self-reported cognitive performance and its minimal relationship to objective data. The need for objective assessment as well as assessment of subjective impressions of cognition is discussed. The use of assessment of performance-based measures of everyday functional skills in major depression, bipolar disorder, and PTSD is another critical development highlighted in the present review. The recent success of pharmacological treatments for cognitive impairments in major depressive disorder (MDD) is promising, yet the need for clinicians to assess and consider bias in their treatment outcome data is essential.
\end{abstract}

Keywords Cognition $\cdot$ Depression $\cdot$ Anxiety $\cdot$ PTSD · Bipolar · MDD

This article is part of the Topical Collection on Mood and Anxiety Disorders

Philip D. Harvey

philipdharvey1@cs.com

1 Department of Psychiatry and Behavioral Sciences, University of Miami Miller School of Medicine, 1120 NW 14th Street, Suite 1450, Miami, FL 33136, USA

2 Research Service, Miami VA Medical Center, Miami, FL, USA

\section{Introduction}

Cognitive impairments, whether subjectively reported, measured more objectively via neurocognitive tests, or measured with performance-based assessment of functional capacity are all well-documented features of mood and anxiety disorders. However, there has been little progress in the treatment of cognitive impairment in these conditions and there is not a full consensus on the most affected domains, the best way to assess them, and the most promising treatment modalities. Additionally, deficits in affective processing for the emotional quality of information will be reviewed so that both what can be conceptualized as "hot" cognition and "cold" cognition (typically assessed with neurocognitive testing) will be represented in the following review. This review will present recent progress with respect to identifying the characteristics of impaired cognition in mood and anxiety disorders and present promising new directions in their assessment and treatment. The role of comorbidities and health-related behaviors in the manifestation, assessment, and treatment of these disorders will also be reviewed. The results of the present review have indicated that most recent and promising new developments have largely been found in the bipolar disorder, major depressive disorder (MDD), and post-traumatic stress disorder (PTSD) literature; thus, those specific illnesses will comprise the focus of the present paper.

The methodology employed for the present review involved a review of electronic databases (supplied by Google and PubMed) from late 2013 though early 2015. Approximately the past 1.5 years was examined for novel research pertaining to cognition in depression and anxiety. The references and bibliographies of the articles were further reviewed and examined for novel research that may have not arisen in 
the initial online searches. Review article, original research, clinical trial, and meta-analysis publication types were included if published in English.

\section{Major Depressive Disorder}

\section{Characteristics}

Cognitive deficits in MDD are widely reported. Both subjective reports and impaired cognitive test performance on measures of attention, executive function, psychomotor speed, psychomotor skills, and memory (verbal and nonverbal episodic) have been routinely found. A recent review of the cognitive effects of pharmacotherapy within MDD on cognition conducted by Keefe et al. [1] brought to light several points worth mentioning about the characteristics of cognitive abnormalities in MDD. First, research to date has not sufficiently resolved the issue as to whether or not cognitive impairments in MDD continue when depressive symptoms resolve or remit. There is considerable evidence that this is the case, but there is less research in this area than in the area of bipolar disorder. Cognitive deficits in depression may exert an additional and negative impact on everyday functioning and coping abilities [1]. Finally, there is little evidence to date that previous generations of antidepressant treatments exerted beneficial effects on cognition. As discussed below, some positive data on cognitive effects exists for recent antidepressants [2]. Although subjective reports of cognitive impairments commonly remit along with depressive symptoms, objective measures of cognitive impairment remain persistently impaired in a number of patients.

A recent review conducted by McIntyre et al. [3] examined the role of cognitive deficits in functional outcomes as well as evidence for promising treatments for cognitive deficits in MDD that we will discuss subsequently. Their review defines cognitive dysfunction as deficits in a cognitive domain as well as negative attentional bias. However, the distinction between subjectively reported cognitive dysfunction and objectively defined impairment using test performance data is an important one that will be subsequently highlighted in the present review. McIntyre et al. [3] noted several major problems with extant research in MDD and cognitive deficits including disparate and wide-ranging age groups, illness severity, illness characteristics (length, severity, subtype, and presence of psychosis), and comorbidities. Most importantly, they highlighted the lack of studies examining MDD patients while in remission, asymptomatic or unmedicated. They also pointed out that early-childhood adversity or trauma is too rarely evaluated in these studies and that there is currently no accepted gold standard for cognitive impairment in MDD. The reliance on deviations from group means in contrast to within-individual differences is likely to result in an underestimation of overall cognitive impairment in MDD [3]. Finally, premorbid cognitive deficits predating the onset of MDD have been studied with less interest than premorbid deficits in schizophrenia, with those studies conducted indicating reduced episodic memory as a possible preclinical and premorbid feature of MDD.

The frequency and duration of depressive episodes have also been associated with poorer neurocognitive outcome, but there is also evidence to support that these deficits can be present in first episodes, so while these deficits may exacerbate impairments, they are not necessary to cause cognitive deficits [3]. Cognitive complaints are common in MDD and are part of the diagnostic criteria. Less research has been dedicated to determining the extent of cognitive deficits in MDD than has in severe mental illness such as schizophrenia and bipolar disorder [3], possibly because of the belief that these deficits are entirely caused by mood states. The impact of cognitive dysfunction on psychosocial function has also been relatively underevaluated $[3,4]$. Based on evidence that cognitive deficits may persist when other symptoms and core features of MDD resolve may indicate the need for a reconceptualization of symptomatic remission in MDD [3], much as functional remission has become a topic of discussion in other conditions such as schizophrenia [5].

Depression is known to result in missed work days and decreased work productivity, which, in addition to the costly and lengthy treatments, contributes to the overall cost and disease burden associated with MDD. McIntyre et al. [6] address the problem of the detrimental effect of MDD on workplace productivity by examining whether cognitive impairment in MDD could potentially be a factor leading to the problem. This study used data from the International Mood Disorders Collaborative Project (IMDCP) with a total of 1861 participants enrolled. Participants with the diagnosis of major depressive disorder were given measures of depression severity, cognitive function, and quality of life. The results of the study showed that workplace performance is predicted to a greater extent by specific cognitive dysfunction than by total depression symptom severity. These results emphasize the importance of finding treatments for the cognitive dysfunction associated with MDD.

Lam et al. [4] hypothesized that the sometimes seemingly resistant functional outcomes (psychosocial and vocational outcomes) in MDD may be mediated by neurocognitive deficits, which also do not universally and reliably improve alongside symptoms. Lam et al. [4] reviewed the literature examining deficits in neuropsychological functioning including attention, learning, working memory, visual and auditory processing, processing speed, motor functioning, and problem solving in the context of MDD. Their review uncovered little support for the mediation of cognition in psychosocial functioning. These findings corroborate those in the schizophrenia literature [7] indicating that while both cognition and 
psychosocial functioning contribute to real-world outcomes, they necessitate separate measurement and represent related but separable aspects of behavior.

\section{Assessment}

One promising new direction in the development of novel assessment approaches in MDD has been the growing focus and recognized utility of using functional capacity measures. A strong example of such measures is the UCSD Performance-Based Skills Assessment (UPSA-B) [8], which is a performance-based measure of functional capacity, whereby patients are asked to perform everyday tasks related to communication and finances. The Communication subtest involves "role-play exercises using an unplugged telephone (e.g., making an emergency call; dialing a number from memory; calling to reschedule a doctor's appointment)." During the Finance subtest, participants read a utility bill, count change, and write and record a check for a bill. The FDA and European regulatory authorities have mandated that functionally relevant outcome measures be used in studies of cognitive enhancement. The UPSA is currently approved by the FDA as suitable for treatment outcome measure in studies of schizophrenia. The UPSA has not only been found to highly correlate with measures of cognition in patients with schizophrenia, but it has also been shown to better predict real-world outcomes. An Institute of Medicine symposium in February of 2015 focused on the use of the UPSA and its variants in major depression, with substantial supportive data presented on its validity and sensitivity to treatment (https://www.iom.edu/ Activities/Research/NeuroForum/2015-FEB-24.aspx). It is important to note that while mood disorders are typically characterized by less severe deficits than those documented in schizophrenia, even healthy controls demonstrate variability on the UPSA, and in a recent MDD treatment study (the results of which are forthcoming) presented at the IOM meeting, no ceiling effects were observed on the UPSAB. Use of a similar functional capacity measure may soon be recognized as an essential addition to the assessment of cognition in all studies of treatments for mood and anxiety disorders.

\section{Cognitive and Emotional Biomarkers}

Day et al. [9] examined the potential cognitive and emotional biomarkers of melancholic depression, which was important because in general, there is a lack of clarity about what differentiates depressed patients with and without melancholic features. Past findings have generally indicated that depressed patients with the melancholic subtype tend to perform more poorly on tasks involving decision-making with increased cognitive load, under time demands, in the areas of set shifting and multi-tasking. The Day et al. [9] study examined many general and emotional cognitive areas of function within patients in both the melancholic and non-melancholic subgroups, as well as healthy controls.

Patients were classified as melancholic or non-melancholic based on scores on the "CORE" rating scale as well as DSM criteria. Participants then completed a battery of tests measuring general and emotional cognitive function. Subtests in the general cognition category included tests measuring motor coordination, response inhibition, sustained attention, information processing, verbal memory, working memory, executive function, and verbal interference. The emotional cognition category included tests of explicit recognition of emotion and implicit face recognition priming. Results of this study showed a significant interaction between the three clinical groups (melancholic, non-melancholic, healthy controls) and the various subtests in the general and the emotional cognition categories. The melancholic group showed poorer performance on all subtests except two. The melancholic group also showed poorer performance on specific domains of testing including information processing speed, decision speed, and cognitive flexibility. The melancholic group also performed worse on the emotional cognition tests; specifically, they displayed a slowed response to happy emotion. Overall, these findings indicate that patients with melancholic major depressive disorder have a specific cognitive performance profile that differs from that of other people with non-melancholic major depressive disorder.

\section{Treatment}

At present, there is no FDA-approved treatment for cognitive impairment in MDD. Keefe et al. [1] reviewed 43 recent research reports which sought to investigate the cognitive effects of previous pharmacotherapies for MDD. The studies included in the review focused on antidepressant agents (SSRIs, serotonin-norepinephrine reuptake inhibitors, and tricyclic inhibitors) as well as other psychotropic agents including antipsychotic agents, hormones, mineralocorticoid receptor modulators, and cognition-enhancing agents such as galantamine. They surmised that while the majority of studies did find a benefit on cognition, there was no consistent pattern and only $12 \%$ of the changes favored active treatment above and beyond those in healthy controls or when placebo was used. Their group further analyzed sample weighted mean effect sizes and found that while verbal memory was found to improve with monotherapy, augmentation therapy yielded the largest treatment effect for visual memory. They concluded that pharmacotherapy does hold promise for the treatment of MDD, with augmentation therapy holding promise to treat the cognitive deficits that persist even once clinical response or remission has resulted from monotherapy. It is important to mention that the pseudo-specificity of cognitive deficits in MDD poses a significant obstacle to the development and 
ultimately the approval of treatments that also benefit cognition. The pseudo-specificity is exacerbated by the lack of correlation between patient self-reports of both cognition and everyday functioning and their own performance on tests of those skills. In contrast, depression and self-report bias are often highly often correlated. This issue of pseudospecificity will be further discussed in greater detail in the review of the bipolar disorder literature.

One recent randomized clinical trial (RCT) and treatment outcome study conducted by Mahableshwarkar et al. [10] examined the new multi-modal antidepressant vortioxetine as a potential treatment for cognitive dysfunction associated with major depressive disorder. The study included $n=602$ subjects diagnosed with MDD that self-reported cognitive dysfunction. Subjects were randomly assigned to an 8-week treatment with either vortioxetine (10 or $20 \mathrm{mg}$ ), placebo, or duloxetine, an older SNRI antidepressant. A battery of cognitive tests measuring different cognitive domains was administered to participants at baseline and the completion of the study. The primary outcome measure of the study was performance on the digit symbol substitution test (DSST) because of previous work showing sensitivity of this measure in MDD has been published $[2,11]$. Vortioxetine treatment led to significant improvement in DSST performance when compared to placebo. Additionally, performance on several other cognitive tasks such as the Trail Making B improved in the vortioxetine group. A path analysis showed that the cognitive improvements due to vortioxetine were independent from the improvements in depressive symptoms. Additionally, while the other antidepressant used in the study (duloxetine) did improve depressive symptoms with equivalent efficacy, when compared to vortioxetine, duloxetine treatment did not improve cognition. Finally, individuals treated with vortioxetine whose depression did not improve with treatment had equivalent cognitive impairments compared to those whose depression did improve, again suggesting a direct cognitive benefit for vortioxetine independent from improvement in depression symptoms. Self-reported improvements in depression were correlated with improvements in mood symptoms but not with objective cognitive test performance. Self-reports of cognitive impairments in depression may have bias components that persist after successful treatment of both depression and cognitive deficits.

Lisdexamfetamine dimesylate (LDX) is currently approved by the FDA for treating ADHD in children, adolescents, and adults. In a recent study, Madhoo et al. [12] examined LDX as a potential treatment for executive dysfunction among people with major depressive disorder. One hundred forty-three adults with mild MDD who were already being treated with an antidepressant were diagnosed with executive dysfunction using the BRIEF-A (Behavior Rating Inventory of Executive Function-Adult Version). Over the study period of 9 weeks, participants were randomly assigned to either LDX or placebo. In addition to being tested with the BRIEF-A at the end of the 9-week treatment, participants also had informants report on their executive function. The researchers found that the LDX group had significant improvements in scores on a self-report measure of executive function. However, they did not exhibit significant improvements on a performance-based measure of executive function. It is important to note that scores on the BRIEF-A were only modestly correlated with performance-based measures of executive function and that informant reports of cognitive deficits and self-reports were also minimally correlated. Thus, subjective response in cognition was correlated with clinical improvement but not substantiated by performance-based assessments. We will return to this issue later.

\section{Metacognitive Therapy for Cognitive Deficits in Depression}

Another recent study [13] examined a type of therapy called metacognitive therapy (MCT) in its ability to improve neuropsychological function among people with major depressive disorder, as compared to cognitive behavior therapy (CBT). The domains of neuropsychological functioning addressed in this study were executive function and attention. While CBT has been found to be a helpful psychotherapy for depression, it does not appear to treat the cognitive impairments that are often associated with depression. MCT aims to treat the cognitive attentional syndrome (CAS) that often accompanies depression. MCT utilizes an attention training technique (ATT) which aims to improve executive function and attention processes in people with depression. Their study treated 48 adults with major depressive disorder (without another comorbid psychiatric disorder) by randomly assigning each participant to receive up to 12 sessions of either CBT or MCT. Participants received a neuropsychological assessment measuring attention and executive functioning. Results showed that by week 12, there was a significant difference between the CBT group and the MCT group on performance on the Groton Maze Learning Task, which measures executive function and spatial problem-solving. These findings suggest that MCT potentially could be a more helpful method of treating the cognitive impairments that accompany depression.

\section{Bipolar Disorder}

\section{Characteristics}

Compared to schizophrenia, cognitive impairments in bipolar disorder are described as less prevalent, manifesting in fewer domains, and overall less severe and, at least in some domains, less persistent [14]. Yet, the nature of the difference in the cognitive performance in bipolar versus schizophrenia samples has been the source of controversy. Current findings 
suggest that impairments observed in bipolar disorders have a similar profile to those in schizophrenia, but with a scale that is midway between healthy controls and schizophrenia [14, 15]. (For further review of a number of relatively recent metaanalyses (published prior to 2013) that closely examine cognitive deficits in bipolar disorder, please refer to our group's previous review [14].) Further, it has been known for an extended period that the impairments seen in patients with bipolar disorder during a mood episode persist in the majority of cases into periods of euthymia.

\section{Assessment}

While most cognitive impairments in mood disorders seem similar to those seen in schizophrenia, there are aspects of affect processing that are also impaired. These impairments are present in both depressed and euphoric states and have the form of excessive processing salience targeted at the emotional nature of information presented. Thus, an effective cognitive assessment of mood disorders, particularly major depression and bipolar disorder, needs to consider these aspects of hot cognition as well as the cold cognition typically assessed with neuropsychological assessments.

In an attempt to develop a cognitive assessment for mood disorders, Keefe et al. [16] tested the reliability and validity of the newly developed Brief Assessment of Cognition in Affective Disorder (BAC-A) in people with bipolar disorder who were currently depressed and participating in a treatment trial. The BAC-A combines tests from the BAC and BACS with other tests that measure the effect of emotional salience on cognition (Affective Processing Test). This study tested people with bipolar disorder along with healthy controls. The results indicated that for the traditional cognitive measures of the BAC-A, patients with bipolar disorder differed in their performance from healthy controls. Additionally, the Affective Processing Test was able to account for additional variance in scores separating the two groups of participants. This indicates that this test is a good measure of cognitive difficulties that arise from affective disorders and that the mood component was important. These findings were confirmed by Bauer et al. [17], who also found that scores on the BAC-A correlated with global measures of everyday disability in people with bipolar disorder.

As previously mentioned, the use of reliable and valid functional capacity measures in the assessment of mood and anxiety disorders is a promising new development. With respect to bipolar disorder specifically, the UPSA has been used in several previous [18] and recent studies [19, 20]. In a large $(n=8140)$ study of patients with bipolar disorder and schizophrenia [19], we found that patients with bipolar disorder manifested substantial impairments on the UPSA-B, with minimal evidence of ceiling effects, and substantial separation from patients with schizophrenia (Cohen's $d=.59$ ) and from healthy standards. These data suggest that future treatment studies of patients with mood disorders will likely add performance-based measures of functional capacity.

Impairments in cognitive, social, and everyday functioning, as well as substantial deficits in the ability to self-assess symptoms and functioning [7], are well documented in schizophrenia. Further, as noted above, self-assessments of cognitive deficits in major depression are also subject to extensive biases, wherein reports of cognitive functioning are actually capturing distress as indexed by major depression symptoms. There has been less attention to this issue in bipolar disorder, particularly in domains of self-assessment of everyday functioning. A recent "pilot study on self-assessment of cognitive, social, and everyday functioning of patients with bipolar depression, comparing self-reports of cognition and everyday functioning to both objective performance-based measures of these domains and impressions of high contact clinicians" was conducted [20]. This study examined selfassessment in a group of 30 bipolar I patients currently experiencing bipolar depression. The patients completed performance-based objective measures of cognition and functional capacity and also provided their self-reported opinions of their everyday functioning and symptoms. High-contact clinicians also provided their impressions of cognitive performance and everyday functioning using identical rating scales. Patient self-reports of both cognition and everyday functioning were uncorrelated with their own performance on tests of cognition and functional capacity, while clinician impressions were correlated with the results of the patient's performancebased assessments. These clinicians were not aware of the scores that the patients obtained, making the correlations that much more important. Also, the substantial biasing effects of current depression on self-reports were quite evident. In contrast to the typical findings for patients with schizophrenia, where patients as a group tend to overestimate their performance, patients with bipolar depression rated themselves as significantly less capable compared to their clinicians' impressions. Also, the correlation between current depression and self-report bias was quite large, with the correlation between self-reported depression and self-reported cognitive deficits at $r=.60$ and correlations between current depression and selfreported everyday functioning ranging from $r=.40$ to $r=.59$.

\section{Comorbidities}

\section{Overweight and Obese Patients with Bipolar Disorder}

Silveira et al. [21] investigated the impact of obesity on cognition in patients in the early phase of bipolar disorder. Participants in this study were 65 patients with bipolar disorder I diagnoses, as well as 35 healthy controls. Participants were classified as either normal weight or overweight and were tested on a cognitive battery at baseline. Cognitive tests 
included tests of processing speed, attention, verbal memory, nonverbal memory, working memory, and executive function. Results from that study indicated that while the bipolar disorder group did have lower scores on cognitive tests than the healthy control group, there were no significant differences in cognition between overweight and normal-weight groups of people in the early stages of bipolar disorder.

A much larger study [22] examined the relationship between obesity and cognition. Cognitive performance was assessed within a sample of 804 adults of full or mixed Ashkenazi Jewish descent with schizophrenia or bipolar disorder, looking for effects of obesity, treated hypertension, and diabetes. Participants were diagnosed with either schizophrenia or bipolar disorder based on DSM-IV criteria. Their BMIs were calculated using self-reported height and weight, and their diagnoses of hypertension and/or diabetes were verified by an examination of the medications they were taking. Neurocognitive ability was measured using a composite score developed by the researchers based on a battery of commonly used neuropsychological tests. The results showed that cognitive ability was significantly different among participants based upon their BMIs (normal, overweight, or obese) in people with bipolar disorder. When looking at the individual cognitive subtests, the researchers found that performance on three of the eight assessments was significantly and negatively associated with BMI in participants with bipolar disorder. These three tests were Digit Symbol, RAVLT, and the continuous performance test. Additionally, a negative association in patients in the bipolar disorder group was found between treatments for hypertension (i.e., medication use) and global cognitive performance, as well as performance on two of the eight subtests (Digit Symbol and Trail Making Test Part A). Higher BMI as well as hypertension and diabetes were associated with lower cognitive functioning in the bipolar disorder patients but not in those with schizophrenia.

Thus, when comparing the results of the two studies above, the impact of obesity may be hypothesized to be greater over time. Patients early in the course of bipolar disorder did not show a relationship between BMI and cognition, while more chronic patients had a very clear relationship. As more chronic patients have a longer treatment history as well, it is important to consider the possible adverse impacts of medication treatments on obesity and subsequently on cognitive impairments. As there is little direct evidence of deleterious effects on cognition of medications used to treat bipolar disorder, the indirect influence of medication on obesity and then subsequently on cognition needs to be considered.

\section{Smoking}

Results have varied in studies examining the cognitive effects of cigarette smoking among people with bipolar disorder and schizophrenia. Some studies have linked smoking to cognitive decline, while others have found nicotine to be positively correlated with cognitive performance. In the same sample described immediately above, Depp et al. [23] recently examined the impact of smoking on both cognitive ability and functional outcome. Smoking status and histories were selfreported and measured with several variables. Functional capacity was assessed using the brief UCSD Performance-Based Skills Assessment (UPSA-B). Functional outcome was measured by having an informant for each participant rate their everyday functioning. The results indicated that current smoking was associated with worse cognitive functioning, compared with the performance of those who were past smokers or who never smoked. This finding persisted after accounting for other variables such as sex, education, and residential independence. However, after adjusting for the variables of negative symptoms and illicit substance use in the past month, the effect was no longer significant. Performance on one cognitive test, the WAIS Digit Symbol, was significantly negatively correlated with smoking before accounting for demographic and symptom variables. The findings were very similar when looking at the effect of smoking on functional capacity and functional outcomes: there was a significant negative relationship between smoking and these variables that did not persist when accounting for symptoms and illicit substance use. These findings indicate a need for future studies to look at these confounding variables of demographics, symptoms, and substance use.

\section{Post-traumatic Stress Disorder}

\section{Characteristics}

Deficits in working memory, learning, sustained attention, and processing speed are frequently observed in patients with PTSD [24, 25]. After all, PTSD has several symptoms that are directly related to abnormal memory functioning, including post-traumatic amnesia and intrusive memories both while awake and asleep. One recent prospective investigation of PTSD, which did utilize performance-based measures of NP, was conducted by Suliman, Stein, and Sedat [26]. They examined the neuropsychological predictors of PTSD in a sample of 131 participants who recently had been involved in road traffic collisions. Their findings indicated that impairment on tests of verbal learning, information processing, executive functioning, and motor speed all predicted PTSD severity even when initial clinical, sociodemographic, and educational factors were accounted for. Thus, cognitive changes may actually predict vulnerability to PTSD as well as result from the PTSD syndrome. These findings are consistent with those from mood disorders and schizophrenia, where alterations in cognitive performance 
prior to onset are viewed as potential markers of vulnerability to the conditions.

\section{Assessment}

Similar to our discussion of new assessment developments in bipolar and MDD populations, the use of functional capacity measures is also a promising new development in the assessment of PTSD. Kaye et al. [24] studied a sample of female patients with PTSD using the UPSA-B in addition to measures of cognition (MCCB) and both clinical and self-reported ratings of PTSD symptoms. UPSA scores were correlated with MCCB scores and lifetime indices of functional and educational attainment. Performance on these measures was moderately impaired compared to healthy norms. However, depression and PTSD scores, both clinician rated and self-reported, as well as self-reported disability, were not correlated with performance on the MCCB or the UPSA-B or with lifetime achievements. Again, these data suggest that in PTSD, like in mood disorders, the impact of distress on subjective self-assessment may be substantial. As much of the assessment of PTSD symptomatology and functioning relies on self-report methods, some caution may be required in the future when designing outcome studies.

\section{Treatment}

Marin et al. [27] examined several potential cognitive enhancers that could be used along with exposure-based therapy for treatment of PTSD. One previous problem with exposurebased therapy is the failure of decreases in fear to the conditioned stimulus to be consolidated into memory and later retrieved. Patients are, therefore, able to learn not to fear a specific stimulus, but after a delay, they fail to remember that they no longer fear this stimulus. For this reason, Marin et al. [27] examined several treatments that could be used along with this type of therapy to perhaps increase cognition and possibly memory. One potential treatment was D-cycloserine. This NMDA receptor agonist has been studied as a potential adjunct treatment along with therapy for phobias for several decades. Studies to date have found mixed results, but nonetheless, treatment with D-cycloserine has been found to be successful in improving treatment outcomes with exposure therapy. Beta blockers, another potential treatment, have been looked at extensively for their potential to treat anxiety and PTSD. Studies have also found that when propranolol is taken immediately following a trauma, rates of PTSD are lower. Additionally, administering propranolol during trauma reactivation has been found to lower physiological response. Endocannabinoids have also been studied for their potential to help with extinction learning. Human preclinical trials have shown that the endocannabinoid system in the brain promotes fear extinction learning. This field is growing in popularity as evidence continues to show that there may be reduced endocannabinoid availability in PTSD.

\section{Conclusions}

There have been several recent developments in the study of altered cognition in mood and anxiety disorders. These include increasing evidence and support regarding the use of performance-based measurement of functional capacity, a domain that is highly linked to both neuropsychological testing performance and everyday functioning. Functional capacity assessment will be a critical aspect of future treatment trials. Also, several pharmacological treatment studies have reported success in the treatment of cognitive impairments in MDD. These studies have also revealed another of the issues recently studied, the tendency of people with mood and anxiety disorders to rate their cognitive performance on the basis of their distress. In contrast to people with psychotic disorders, current ability and future potential seem to be underrated in these individuals. Thus, objective assessment in treatment trials will be critical, but continuing to assess impressions of cognitive performance will be critical for understanding of the patient perspective. Clinicians will need to understand these biases, however, as more effective treatments for cognition become available.

\section{Compliance with Ethics Guidelines}

Conflict of Interest Philip Harvey has worked as a consultant for AbbVie, Boehringer Ingelheim, Forest Labs, Forum Pharma, Genentech, Lundbeck, Otsuka America, Roche, Sanofi, Sunovion, and Takeda Pharma. Harvey received contracted research support from Genentech. Sara Kaplan has no disclosures relevant to this work. Felicia has received consulting fees from Clintara Inc. during the past year.

Human and Animal Rights and Informed Consent This article does not contain any studies with human or animal subjects performed by the authors.

\section{References}

1. Keefe RS, McClintock SM, Roth RM, Doraiswamy PM, Tiger S, Madhoo M. Cognitive effects of pharmacotherapy for major depressive disorder: a systematic review. J Clin Psychiatry. 2014;75:864-76.

2. McIntyre RS, Lophaven S, Olsen CK. A randomized, double-blind, placebo-controlled study of vortioxetine on cognitive function in depressed adults. Int J Neuropsychopharmacol. 2014;17:1557-67.

3. Mcintyre RS, Cha DS, Soczynska JK, Woldeyohannes HO, Gallaugher LA, Kudlow P, et al. Cognitive deficits and functional outcomes in major depressive disorder: determinants, substrates, and treatment interventions. Depress Anxiety. 2013;30:515-27.

4. Lam RW, Kennedy SH, McIntyre RS, Khullar A. Cognitive dysfunction in major depressive disorder: effects on psychosocial 
functioning and implications for treatment. Can J Psychiatry. 2014;59:649-54.

5. Harvey PD, Bellack AS. Toward a terminology of functional recovery in schizophrenia: is functional remission a viable concept? Schizophr Bull. 2009;35:300-6.

6. McIntyre RS, Soczynska JZ, Woldeyohannes HO, Alsuwaidan MT, Cha DS, Carvalho AF, et al. The impact of cognitive impairment on perceived workforce performance: results from the international mood disorders collaborative project. Compr Psychiatry. 2015;56: 279-82.

7. Harvey PD, Pinkham AE. Impaired self-assessment in schizophrenia: why patients misjudge their cognition and functioning. Curr Psychiatry. 2015;14:53-9.

8. Mausbach BT, Harvey PD, Goldman SR, Jeste DV, Patterson TL. Development of a brief scale of everyday functioning in persons with serious mental illness. Schizophr Bull. 2007;33:1364-72.

9. Day CV, Gatt JM, Etkin A, DeBattista C, Schatzberg AF, Williams LM. Cognitive and emotional biomarkers of melancholic depression: an iSPOT-D report. J Affect Disord. 2015;176:141-50.

10. Mahableshwarkar AR, Zajecka J, Jacobson W, Chen Y, Keefe Richard SE. A randomized, placebo-controlled, active-reference, double-blind, flexible-dose study of the efficacy of vortioxetine on cognitive function in major depressive disorder. Neuropsychopharmacology. 2015;1-13.

11. Katona C, Hansen T, Olsen CK. A randomized, double-blind, placebo-controlled, duloxetine-referenced, fixed-dose study comparing the efficacy and safety of Lu AA21004 in elderly patients with major depressive disorder. Int Clin Psychopharmacol. 2012;27: 215-23.

12. Madhoo M, Keefe Richard SE, Roth RM, Sambunaris A, Wu J, Trivedi $\mathrm{MH}$, et al. Lisdexamfetamine dimesylate augmentation in adults with persistent executive dysfunction after partial or full remission of major depressive disorder. Neuropsychopharmacology. 2014;39:1388-98.

13. Groves SJ, Porter RJ, Jordan J, Knight R, Carter JD, McIntosh Virginia VW, Fernando K, Frampton Christopher MA, Mulder RT, Lacey C, Joyce PR. Changes in neuropsychological function after treatment with metacognitive therapy or cognitive behavior therapy for depression. Depress Anxiety. 2015:1-8.

14. Harvey PD, Wingo AP, Burdick KE, Baldessarini RJ. Cognition and disability in bipolar disorder: lessons from schizophrenia research. Bipolar Disord. 2010;12:364-75.

15. Bowie CR, Holshausen K, Gupta M. Comparative impairments across schizophrenia and bipolar disorder. In: Harvey PD, editor. Cognitive impairments in schizophrenia. New York: Cambridge University Press; 2013. p. 50-68.

16. Keefe Richard SE, Fox KH, Davis VG, Kennel C, Walker TM, Burdick KE, et al. The brief assessment of cognition in affective disorders (BAC-A): performance of patients with bipolar depression and healthy controls. J Affect Disord. 2014;166:86-92.

17. Bauer IE, Keefe RS, Sanches M, Suchting R, Green CE, Soares JC. Evaluation of cognitive function in bipolar disorder using the Brief Assessment of Cognition in Affective Disorders (BAC-A). J Psychiatry Res. 2015;60:80-6.

18. Bowie CR, Depp C, McGrath JA, Wolyniec P, Mausbach BT, Thornquist $\mathrm{MH}$, et al. Prediction of real world functional disability in chronic mental disorders: a comparison of schizophrenia and bipolar disorder. Am J Psychiatry. 2010;167:1116-24.

19. Harvey PD, Siever LJ, Huang GD, Muralidhar S, Zhao H, Miller P, et al. The genetics of functional disability in schizophrenia and bipolar illness: methods and initial results for VA comparative study \#572. Am J Med Genet Part B. 2014;165B:381-9.

20. Harvey PD, Paschall G, Depp C. Factors influencing selfassessment of cognition and functioning in bipolar disorder: a preliminary study. Cogn Neuropsychiatry. 2015;20:361-71.

21. Silveira LE, Kozicky J, Muralidharan K, Bucker J, Torres IJ, Bond DJ, et al. Neurocognitive functioning in overweight and obese patients with bipolar disorder: data from the systematic treatment optimization program for early mania (STOP-EM). Can J Psychiatry. 2014;59:639-48.

22. Depp CA, Strassnig M, Mausbach BT, Bowie CR, Wolyniec P, Thornquist MH, et al. Association of obesity and treated hypertension and diabetes with cognitive ability in bipolar disorder and schizophrenia. Bipolar Disord. 2014;16:422-31.

23. Depp CA, Bowie CR, Mausbach BT, Wolyniec P, Thornquist MH, Luke JR, McGrath JA, Pulver AE, Patterson TL, Harvey PD. Current smoking is associated with worse cognitive and adaptive functioning in serious mental illness. Acta Psychiatr Scand. 2015: $1-9$.

24. Kaye JL, Dunlop BW, Iosifescu DV, Mathew SJ, Kelley ME, Harvey PD. Cognition, functional capacity, and self-reported disability in women with posttraumatic stress disorder: examining the convergence of performance-based measures and self-reports. J Psychiatr Res. 2014;57:51-7.

25. Twamley EW, Allard CB, Thorp SR, Norman SB, Hami Cissel S, Hughes Berardi K, et al. Cognitive impairment and functioning in PTSD related to intimate partner violence. J Int Neuropsychol Soc. 2009;15:879-97.

26. Suliman S, Stein DJ, Seedat S. Clinical and neuropsychological predictors of posttraumatic stress disorder. Medicine (Baltimore). 2014;93:e113.

27. Marin MF, Lonak SF, Milad MR. Augmentation of evidence-based psychotherapy for PTSD with cognitive enhancers. Curr Psychiatry Rep. 2015;17:582. 